\title{
Propuesta de prevención primaria de la anorexia y bulimia en la infancia
}

\author{
Patricia Correa, Patricia Carbia, Irene Crestar, María de Coo, Mª Ángeles Diz, Tamara Fernández
}

\begin{abstract}
Resumen
El incremento de los casos de trastornos de la conducta alimenticia en la infancia plantea la necesidad de crear una herramienta didáctica y orientativa para su prevención. Se busca trabajar conjuntamente con todos los agentes socializadores que afectan a la vida del niño: familiar, escolar y comunitario. En este trabajo se pretende ofrecer una serie de puntos clave y actividades en el aula, para la prevención de la aparición de los trastornos de alimentación más frecuentes (anorexia y bulimia), partiendo y adaptando las tareas a las características propias de la edad infantil.
\end{abstract}

Palabras clave: Prevención, anorexia, bulimia, infancia, trastornos de la conducta alimenticia

\section{Introducción}

Entre los trastornos alimenticios que más afectan a la población destacan la anorexia y la bulimia, socialmente ligados a la adolescencia. Sin embargo la insatisfacción corporal se puede iniciar en la infancia, aumentando de forma progresiva durante la pubertad y adolescencia (Ferreiro, Seoane, y Senra, 2014).

Por ello es importante trabajar estos aspectos desde edades tempranas, evitando así el efecto negativo de ciertos factores sobre aquellos individuos que presentan una mayor vulnerabilidad.

Waddell, Godderis, Schwatz y Garland (2005) identifican cuatro categorías donde englobar los distintos factores de riesgo: individual (perfeccionismo y dietas), familiar (antecedentes en cuanto a problemas alimenticios), cultural (sexo femenino, comparaciones sociales y burlas...) y las características biológicas. Esta clasificación se puede complementar con lo dicho por López y Sallés (2005), quienes identifican los siguientes factores: baja autoestima, nivel alto de autoexigencia, miedo a madurar o crecer y la autoimposición de dietas como factores personales. Finalmente, la realización de ciertas actividades de riesgo, el modelo social o influencia de los medios de comunicación dentro de la categoría cultural.

Existen numerosos programas orientados a la prevención de estos trastornos. Los de mayor éxito son aquellos que emplean técnicas cognitivo-conductuales y de alfabetización mediática con el fin de cambiar actitudes y conductas no saludables (Waddell, Godderis, Schwatz y Garland, 2005). Stice, Shaw y Marti (2007) identifican que existen ciertas características que favorecen el éxito de los programas de prevención entre las que destacan:

- El estatus de riesgo en los participantes: los programas selectivos producen mayores efectos de la intervención.

- El sexo de los participantes: programas dirigidos exclusivamente a mujeres tienen mejores resultados.
- La edad de los participantes: los efectos son mayores para aquellos individuos mayores de quince años.

-El formato del programa: los mayores efectos se producen en aquellos programas interactivos y que enseñan nuevas habilidades (Larimer \& Cronce 2002).

-El tipo de intervención: en este ámbito destacan aquellos programas que son llevados a cabo por intervencionistas preparados. Debido a que aquellas personas pertenecientes al centro tienen otras responsabilidades que pueden hacer que no se entreguen plenamente a los programas.

-El número de las sesiones: los resultados de las multisesiones pueden producir mayores efectos porque son útiles para que los participantes reflexionen sobre el material dado. Los periodos entre sesiones permiten poner en práctica nuevas habilidades y regresar al grupo para pedir consejo sobre la resolución de problemas.

-El contenido del programa: los programas más efectivos son aquellos de contenido inductivo- disonante los cuales destacan en factores como la internalización del ideal de delgadez, la insatisfacción corporal, la dieta o las patologías alimenticias.

El hecho de que se trate de un recurso para trabajar en las aulas de educación infantil y primaria y en el hogar hace que no se tengan en cuenta ciertas características de los programas de mayor éxito como el sexo o la edad. Haría que la propuesta fuese imposible de aplicar en un aula con alumnado de ambos sexos puesto que implicaría la exclusión de parte del mismo a la hora de realizar las actividades, por ejemplo.

Debido a la existencia de poca literatura sobre la prevención de este tipo de trastornos en la infancia, se elabora la propuesta presentada, donde se plantean actividades centradas en reducir las características que hacen más vulnerable al alumnado. Para ello se llevará a cabo la creación de un recurso propio que permitirá reducir la incidencia de los factores de riesgo presentes en el entorno inmediato del niño o niña.

\section{Método}

Se trata de una propuesta que surge del ámbito universitario $\mathrm{y}$, tras llevar a cabo una revisión bibliográfica, se pretende realizar un programa de prevención de los trastornos alimenticios en la edad infantil. Se presentan dos propuestas, una dirigida a la familia y otra a la escuela, donde se recoge la información más importante para ambos contextos que son los más próximos al alumnado. Aunque dichos datos son muy semejantes, presentan diferencias por ser más específicos de un determinado ámbito.

Tras analizar la literatura, nos encontramos con una serie de temas que son más relevantes para llevar a cabo una buena prevención: alimentación saludable, cánones de belleza, autoestima y autoconcepto, aceptación de la diversidad y deporte. 
En cuanto a la estética del proyecto, hemos ideado un formato atractivo y manejable para todos los destinatarios y destinatarias. Se trata pues de dos muñecas recortables, niño y niña, acompañadas de su ropa. Sobre dichas muñecas iremos colocando las diferentes prendas que necesitemos en cada momento según lo que se quiera trabajar, donde llevan escritos los datos más relevantes.

El recortable estará hecho de un material flexible, adaptado y apropiado para las edades de los niños y niñas a los que va dirigido. Se trata de cartulinas blancas DIN-A 3 plastificadas sobre las que se han impreso a color los dos muñecos. Las prendas irán sujetas al cuerpo por medio de solapas y de belcros permitiendo así que se intercambien cuando sea necesario según el tema que se quiera trabajar o tratar. El diseño de la ropa y la elección de los colores buscan no corresponderse con los estereotipos sociales, pudiendo así intercambiarse las prendas entre ambos muñecos.

La utilización de los recursos es la siguiente: se busca que los padres lean el folleto $\mathrm{y}$, posteriormente, sea recortado con los pequeños. De esta manera, les quedarán las muñecas recortables listas para poder jugar con ellas. Mientras que en clase, los muñecos estarán colgados en la pared para que, cada día, el niño que sea el protagonista de esa semana, coja la ropa correspondiente y se la ponga a los muñecos. Una vez leída, se realizará dicha actividad y la ropa quedará puesta en los muñecos hasta el siguiente día.

\section{Resultados}

El resultado del trabajo se recoge en una propuesta de actividades para prevenir conductas que deriven en anorexia y bulimia. Para el profesorado sería necesario ampliar el material una unidad didáctica, en la cual tienen las actividades a realizar en cada momento, con sus objetivos, orientaciones didácticas, criterios de evaluación y materiales necesarios. Finalmente, a los padres, también se les ofrece un material en forma de folleto para que puedan tener a mano información sobre los trastornos, así como algunos consejos para ayudar a su prevención.

Los materiales elaborados se materializan en 3 grupos de materiales: Cuento con muñecos recortables para niños, explicación de actividades complementarias para profesores, y folleto para padres:

\section{Cuento con muñecos recortables:}

Se plantea un cuento interactivo, asociado a una serie de actividades que trabajará el profesor en la unidad didáctica que se plantea a continuación. Presentamos el cuento con una serie de indicadores numéricos que están asociados a las actividades que planteará el profesor mientras trabajan con el cuento (en el apartado B se explicarán las actividades asociadas).

\section{Cuento:}

"Un día, durante el recreo, nuestros amigos se preguntan qué ingredientes llevan esas galletas tan ricas que están comiendo y si son buenas para crecer. Al llegar a clase, le preguntan al profe, quien les habla de la "Pirámide de los alimentos", y entre todos van nombrando numerosos alimentos que eran sanos y otros que no tanto. (1)

Al día siguiente el profe les ha llevado un montón de comida itodos los tipos de pan, de cereales, de pasta, de aceite, de leche, de frutas, de verduras...! Les pide que prueben un poco de cada y después, entre todos, deben decir en qué sitio de la pirámide lo colocarían. Para acabar el día, todos y todas deberán hacer una manualidad sobre estos alimentos, y colocarlo en su lugar de la que será su propia pirámide de los alimentos. Hoy han aprendido que todos estos alimentos deben tomarlos cada día para poder crecer, aunque esto no es lo único, ya que deben de hacer alguna actividad para que su cuerpo esté en forma. A partir de entonces, en el cole harán alguna actividad o juego cada día para empezar las clases con energía.

A la semana siguiente, cuando llegaron a clase encontraron un montón de carnes, huevos, pescados... A muchos de sus compañeros y compañeras no les gustaban aquellos alimentos, o eso creían, porque en cuanto los probaron, descubrieron que estaban buenísimos y que, además, eran importantísimos para crecer. Tras añadirlos a su propia pirámide descubrieron que era muy importante hacer algo de actividad física $y$, así aprovecharon las clases de educación física para descubrir y aprender nuevos deportes. Además con el tiempo se dieron cuenta de que no todos eran igual de buenos y de buenas en todo $y$ que, obviamente, no todos ni todas preferían el mismo deporte ¿Creéis que en vuestra clase pasará lo mismo? ¡Vamos a descubrirlo!

La gran sorpresa llegó a la tercera cuando encontraron la clase llena de dulces iEste piso sí que les iba a gustar! Pero tras hablar con el profe aprendieron que no deben tomarlos en exceso porque no son sanos, alguno de vez en cuando sí, pero no todos los días porque les pueden hacer daño. Y además hay otras actividades que no deben hacer todos los días como ver la televisión, por ejemplo, así que decidieron cambiar los ratos que la veían en casa, por otras actividades como leer, pintar o pasear con su familia, por ejemplo. Empezaron a aprender a disfrutar de ese ratito que tenían y a descubrir que había muchas actividades que no les gustaban a todos y a todas igual y a disfrutar de las que les gustaban a sus compañeros y compañeras.

Ya conocían la pirámide, y pensaron en todas las comidas típicas de Galicia. Con ayuda del profesor, las clasificaron por categorías, según los ingredientes principales que llevaban. Como ya habían probado muchos de ellos, solo tuvieron que recordarlos $y$ buscarlos en la propia pirámide. Los protagonistas de la semana se enfundaron el gorro de cocinero $y$ cocinera $y$, con ayuda de todos y de todas, fueron cogiendo los ingredientes de la pirámide para hacer un cocido a su manera y los fueron colocando en un plato. ¡Ya tienen su cocido hecho! Pero... ¿Es bueno comer esto todos los días? ¿Vosotros que pensáis? (2)

Un día antes de las vacaciones, una compañera comentó que se iba a ir de viaje. Hablando de los países que conocían, descubrieron que no sabían 
casi nada y les picó la curiosidad. Decidieron usar cada semana para conocer un país diferente, sus comidas y los alimentos más usados; además podrían aprovechar y saber cual era el deporte que más gustaba. (3)

Cuando acabaron de ver los distintos países se sorprendieron al ver que lo que más les llamara la atención había sido las diferencias entre las personas desde su aspecto físico a su vestimenta. Así se dieron cuenta de que había algo que les gustaba de todos y entre todas y todos fueron diciendo qué era lo que más les gustaba de esas personas.

Después el profe les dió un montón de partes del cuerpo recortadas de dibujos y de fotos, para que, en pequeños grupos escogieran las que más les gustaban y que hiciesen una persona. Vieron que las personas hechas con las partes del cuerpo "perfectas" eran raras con lo que llegaron a la conclusión de que nadie es perfecto y que eso es bueno. (4)

Decidieron que iban a empezar a ver a la gente de un modo diferente, apreciando todas sus cosas buenas $e$ intentando reducir las cosas no tan buenas que pudieran tener. Para ello, escogerían cada semana a una o un protagonista que tendría un pequeño cuaderno, sobre el que escribirían o dibujarían todos y todas algo bueno acerca de su dueño o dueña. Este cuaderno les ayudaría a sonreír los días que estuviesen un poquito más tristes. (5)”

\section{Explicación de actividades complementarias para} profesores:

Durante el trabajo con el cuento el profesor trabajará las distintas actividades que ayudarán a profundizar en los distintos aspectos que queremos trabajar con este material. Es importante que las familias estén al tanto de lo que se trabaja en el aula para poder continuar con esta labor en sus casas y así darle una mayor uniformidad a la temática.

(1) Nombre: Actividad pirámide.

Objetivos: asimilar y relacionar la alimentación sana con la actividad física; que alimentos son relevantes para llevar una vida saludable y cuáles serían los menos indicativos para una vida sana.

Descripción: los niños y niñas probarán la variedad de alimentos pertenecientes a los distintos pisos de la pirámide, la cual será muy simple, contando solo con tres pisos, ya que está destinada a niños y niñas de edad temprana. Además se debe llevar a cabo una pequeña reflexión sobre la vida saludable y el deporte cuando se hable de cada piso de la pirámide. Una vez finalizada la discusión sobre cada alimento, se realizará una manualidad sobre dicho grupo de alimentos y se colocará en la fila correspondiente de la pirámide. Los alimentos los podrán elaborar con distintos materiales. Esta pirámide la harán los alumnos y alumnas a modo de bandeja o de mural, dependiendo de lo que les permitan las manualidades realizadas, para poder colocar los alimentos cada semana.

Temporalización: Un día por semana durante cuatro semanas.

Recursos:
Materiales: Papel, materiales que decida el profesor o profesora para hacer las manualidades (Goma eva, cartulina, plastilina, recortes de revistas de supermercados,...)

\section{Orientaciones didácticas:}

o Se busca que las actividades tengan continuidad y es recomendable que estas dinámicas se continúen a lo largo de todo el curso y en casa

o Se busca que los niños y niñas disfruten de los ratos ya establecidos y que los vean de otro modo, por ello se les darán mayor importancia.

\section{(2) Nombre: Conociendo nuestra gastronomía}

Objetivos: desarrollar el pensamiento crítico de los niños y niñas acerca de lo que comen con frecuencia.

Descripción: Los alumnos dirán comidas típicas de Galicia y luego, en pequeños grupos, analizarán los ingredientes de cada una buscando su lugar en la pirámide. Después, cada grupo decidirá una comida para preparar. Para ello cogerán los ingredientes necesarios de nuestra pirámide y los colocarán en un plato. Finalmente, y una vez todos hayan acabado, un miembro de cada grupo explicará su plato.

Temporalización: Una sesión de hora y media

\section{Recursos:}

- Materiales: plato de plástico uno por grupo, alimentos

\section{Orientaciones didácticas:}

o Se puede hacer que la selección de los alimentos sea como una visita al supermercado así se hace más atractiva la actividad y aprenden habilidades sociales y planificación (deben pensar los ingredientes, hacer la lista, elegir entre todos, calcular el precio...)

(3) Nombre: Investigando por el mundo

Objetivos: promover y relacionar de forma dinámica cada cultura con el país al que pertenece

Descripción: Cada semana conocerán un país diferente que elegirán todos juntos previamente. De cada país investigarán sus costumbres alimenticias, las comidas más típicas, su deporte más conocido e incluso las características físicas (si las hay) que son prototípicas de los ciudadanos del mismo.

Temporalización: Una sesión por semana, durante 10 semanas

\section{Recursos:}

- Materiales: mapa del mundo, videos sobre estas culturas.

Orientaciones didácticas:

o Se puede iniciar cada sesión con un video didáctico acerca de la región que se trabaje esa semana.

o Sobre todo debemos tratar la dieta propia de ese país, el deporte más practicado y los rasgos físicos más característicos de la población.

o Hay que tener en cuenta que algunos alimentos y platos son más sencillos de trabajar que otros y por lo tanto más adecuados para los más pequeños

o Los deportes, sería interesante trabajarlos en educación física, dentro de las posibilidades que ofrezca el centro.

(4) Nombre: Puzzle loco

Objetivos: Ser conscientes de que todos somos diferentes y que cada uno de nosotros tiene características personales diferentes que nos hacen únicos e irrepetibles 
Descripción: Se debe dividir el aula en pequeños grupos y entregar partes del cuerpo recortadas de dibujos y fotos para que escojan aquellas que más les gusten y hagan a una persona.

Temporalización: 2 horas aproximadamente

Recursos:

- Materiales: Recortes de dibujos y fotos, folios, pegamentos y tijeras.

Orientaciones didácticas:

o El profesor o profesora debe ayudar en la reflexión de la actividad para que comprendan el objetivo de la misma.

o El trabajo realizado se puede exponer en la clase colgado.

o Dependiendo de la edad, los materiales deberán estar ya recortados o no.

(5) Nombre: Don y doña Cuaderno

Objetivos: Mejorar la autoestima de todos los alumnos y alumnas.

Descripción: Cada semana, se escoge a un niño o a una niña para ser el protagonista de ese período de tiempo. Éste deberá colocar la prenda al muñeco recortable según la parte de la historia que toque tratar y ayudará en diferentes tareas. Por otro lado, contará con un pequeño cuaderno, en el cual escribirán todo aquello que se les da mejor y lo que se les da peor. Además, sus compañeros y compañeras al final de la semana, también podrán participar dibujando o escribiendo cosas positivas que tiene el protagonista o la protagonista en el mismo cuaderno.

Temporalización: Durante todo el transcurso de la propuesta de actividades.

\section{Recursos:}

- Materiales: Cuaderno, lápices, pinturas,...

Orientaciones didácticas:

Pueden revisar el cuaderno tantas veces como quieran para mejorar su autoestima, ya que todos tenemos cosas buenas.

\section{Folleto para padres}

Es importante completar este trabajo de aula con un folleto informativo para padres, para ayudarles a comprender los trastornos de alimentación, y cómo la prevención juega un papel fundamental en su posible posterior aparición. Los contenidos que se incluirían en dicho folleto informativo son los siguientes:

\section{¿Cuales son los trastornos alimentarios más frecuentes?}

Anorexia: Trastorno de la conducta alimenticia que se debe a la consumición de escasa cantidad de alimentos. Estas personas continúan adelante con sus dietas extremas, poniendo en peligro su vida, ya que se perciben con sobrepeso.

Bulimia: Trastorno psicológico de la conducta alimenticia caracterizado por una ingesta exagerada e insaciable de alimentos. Esto concluye devolviendo los alimentos, abusando de laxantes y diuréticos, o llevando a cabo dietas restrictivas intermitentes hasta llegar a realizar esto de manera.

\section{¿Qué factores son importantes para su desarrollo?}

Individuales: baja autoestima, nivel alto de - autoexigencia y perfeccionismo, miedo a madurar o crecer y la imposición de dietas que se prolongan en el tiempo.

- Familiar: modelo familiar o existencia de otros trastornos alimenticios como la obesidad.

- Cultural: sexo femenino, comparaciones sociales y burlas, realización de ciertas actividades de riesgo como deportes a nivel profesional, el modelo social o influencia de los medios de comunicación

- Características biológicas: tendencia a engordar

\section{¿Qué podemos hacer?}

- Prevenir este tipo de trastornos ya desde edades tempranas.

- Darle importancia sin considerarlas enfermedades de moda.

- Trabajar estrategias que mejoren las características psicológicas que los hacen más vulnerables y les ayuden a aceptarse.

- Mostrar indiferencia ante la negativa de comer para llamar la atención, dejando que coman por su propio deseo

- Reforzar la construcción de roles, la autoestima y fomentar valores y ser modelo para conseguir una vida saludable.

- Orientar sus intereses y habilidades hacia actividades que permitan la realización personal.

- Educar en una alimentación saludable y asociar las comidas familiares con momentos divertidos.

- Fomentar su autoconcepto y su autoestima, mediante valoraciones positivas,...

\section{¿Dónde puedo pedir ayuda?}

Ante la mínima sospecha de que tu niño o niña pueda sufrir uno de estos trastornos debes consultar con tu pediatra, él te informará de los recursos más adecuados, y os pondrán en contacto con el especialista oportuno.

También existen asociaciones que os pueden ayudar a entender mejor estos trastornos y cómo trabajarlo.

\section{Discusión}

Por último, cabe destacar que se trata de un trabajo puramente teórico, debido a la imposibilidad de ponerlo en práctica con sujetos hasta el momento, con la intención de que otros profesionales lo puedan aplicar y llevar a cabo, tanto en el aula como en el seno familiar.

Con esta propuesta pretendemos transmitir a las familias y a la comunidad educativa, que es posible que este tipo de trastornos, o alguna de sus características, aparezcan en la edad infantil. Esto es debido a que estas edades están un poco abandonadas en relación a este tema y a su investigación y, además, también queremos destacar la importancia de empezar a trabajar los factores de riesgo en los primeros años de vida para evitar estos trastornos de manera temprana y reducir las posibilidades de desarrollarlos en otras edades.

Factores como las dietas, los medios de comunicación, la presencia de otros trastornos o la sociedad son clave en la futura aparición de problemas en la alimentación y es por eso que debemos comenzar a trabajarlo tanto en el hogar como en la escuela, previniendo así mediante la colaboración y el trabajo diario su aparición e intentando evitar que el número de 
casos continúe aumentando. La efectividad será mayor cuanto más se trabajen estos aspectos, sobre todo si se prolongan a lo largo del periodo escolar.

Otro punto importante y decisivo es la coordinación e inclinación hacia la prevención por parte de los dos ámbitos. Si bien esta coordinación es inexistente, el resultado que queremos conseguir difícilmente se efectuará y posteriormente puede que estas pautas de prevención deriven en un efecto positivo o un efectivo negativo en el alumnado.

\section{Referencias}

Ferreiro, F., Seoane, G., \& Senra, C. (2014). Toward understanding the role of body dissatisfaction in the gender differences in depressive symptoms and disordered eating: A longitudinal study during adolescence. Journal of adolescence, 37(1), 73-84.
Stice, E., Shaw, H., \& Marti, C. N. (2007). A metaanalytic review of eating disorder prevention programs: encouraging findings. Annu. Rev. Clin. Psychol.,3, 207-231.

Waddell, C., Godderis, R., Schwartz, C., \& Garland, O. (2005). Preventing and treating eating disorders in children and youth. A research report prepared for the British Columbia Ministry of Children and Family Development

\section{Agradecimientos}

Por confiar en nosotras desde el principio, motivarnos a mejorar constantemente y ayudarnos en la realización de este proyecto. Gracias Rocío Gómez Juncal. 